\title{
Origin of charge density wave formation in insulators from a high resolution photoemission study of $\mathrm{BaIrO}_{3}$
}

\author{
Kalobaran Maiti, Ravi Shankar Singh, V.R.R. Medicherla, S. Rayaprol, and E.V. Sampathkumaran \\ Department of Condensed Matter Physics and Materials' Science, \\ Tata Institute of Fundamental Research, Homi Bhabha Road, Colaba, Mumbai - 400 005, INDIA
}

(Dated: June 28, 2018)

\begin{abstract}
We investigate the origin of charge density wave (CDW) formation in insulators by studying $\mathrm{BaIrO}_{3}$ using high resolution $(1.4 \mathrm{meV})$ photoemission spectroscopy. The spectra reveal the existence of localized density of states at the Fermi level, $E_{F}$, in the vicinity of room temperature. These localized states are found to vanish as the temperature is lowered thereby, opening a soft gap at $E_{F}$, as a consequence of CDW transition. In addition, the energy dependence of the spectral density of states reveals the importance of magnetic interactions, rather than well-known Coulomb repulsion effect, in determining the electronic structure thereby implying a close relationship between ferromagnetism and CDW observed in this compound. Also, Ba core level spectra surprisingly exhibit an unusual behavior prior to CDW transition.
\end{abstract}

PACS numbers: 71.45.Lr, 71.23.-k, 79.60.-i, 71.27.+a

The investigation of charge density wave (CDW) and its influence on the properties of materials has been the subject of intense research for many decades now. Such a phenomenon is often observed in low-dimensional metallic systems due to the spatial modulation of the conduction electron density, the periodicity of which is not commensurate with the unit-cell. As a consequence, a gap opens up in the single particle excitation spectrum of such materials below the transition temperature. Clearly, CDW is expected only for metallic systems. Interestingly, $\mathrm{BaIrO}_{3}$, an insulating material, exhibits CDW transition at about $175 \mathrm{~K} \mathrm{[1]}$. As such an unusual observation is likely to evoke considerable interest, it is important to understand the origin of the CDW anomaly in such insulating materials.

$\mathrm{BaIrO}_{3}$ exhibits many interesting crystallographic and magnetic anomalies [1, 2, 3, 4, 5]. The crystal structure is monoclinic (space group $\mathrm{C} 2 / \mathrm{m}$ ) and consists of $\operatorname{Ir}_{3} \mathrm{O}_{12}$ trimers, where the $\mathrm{IrO}_{6}$ octahedra are face shared. Intertrimer link occurs by corner-sharing to form columns parallel to $c$-axis, as shown in the inset of Fig. 1. It appears [4] that the trimers are marginally tilted with respect to each other and thus a moment-containing chain runs parallel to $c$-axis. Quasi-one-dimensional nature of this compound is evident from the anisotropic transport behavior [1]. The complexity of the structure results in twisting and buckling of the trimers, and in multiplicity of Ir-O and Ba-O bond distances, thereby creating 4 types of $\mathrm{Ir}$ and 3 types of $\mathrm{Ba}$ [3, 6]. A finding unique to this compound is that ferromagnetism sets in at the same temperature at which CDW forms! Thus, it is fascinating that there is an intimate relationship between magnetism and lattice. Therefore, there is an urgent need to throw more light on this relationship. Many other interesting properties are exhibited by this compound, for instance, very low magnetic moment, additional transitions around 80 and $26 \mathrm{~K}$, non-metallicity despite very short Ir-Ir distances etc. All these anomalies reveal that $\mathrm{BaIrO}_{3}$ is truly an exotic material, warranting further urgent investigations.

In this letter, we provide experimental evidence for the fact that there are indeed electronic states at the Fermi level, $E_{F}$, which are localized presumably due to crystallographic features like disorder and distortions, and that the CDW evolves due to these localized electronic states. While the existence of such localized states forming a 'pseudogap' at $E_{F}$ has been predicted long ago 7, , 8], it is the high resolution employed in the present study that enables us to show directly the presence of such states and to track its temperature dependence to understand the CDW in insulators. $\left|E-E_{F}\right|^{3 / 2}$-dependence of the spectral density of states (SDOS) emphasizes the role of magnetism in determining the electronic structure. In addition, we observe significant changes in Ba core level spectra prior to CDW transition suggesting an importance of $\mathrm{Ba}-\mathrm{O}$ covalency in its electronic properties.

The sample was prepared by a conventional solid state reaction method in the polycrystalline form using ultrahigh pure ingredients $\left(\mathrm{BaCO}_{3}\right.$ and Ir metal powders). In order to achieve large grain size and stoichiometry, the final product was pelletized and sintered at a rather high temperature $\left(1000{ }^{\circ} \mathrm{C}\right)$ for more than 2 days and furnacecooled (rather than quenching) which ensures the oxygen stoichiometry close to 3 5]. The $x$-ray diffraction pattern exhibits single phase without any signature of impurity and the lattice constants $(a=10.015 \AA, b=5.748 \AA$, $c=15.157 \AA$ and $\beta=103.27^{\circ}$ ) are found to be in excellent agreement with those reported for single crystals [1]. In addition, we performed electrical resistivity measurements by a conventional four-probe method and establish the presence of a CDW transition at $183 \mathrm{~K}$ from an insulating phase; the onset of a ferromagnetic transition at the same temperature is observed by dc magnetic susceptibility measurements taken in the presence 
of a magnetic field of $5 \mathrm{kOe}$. Somewhat higher transition temperature observed in this compound compared to the single crystals 1] ensures good quality of the sample. Photoemission measurements were performed using a Gammadata Scienta analyzer, SES2002 at a base pressure of $4 \times 10^{-11}$ torr. The experimental resolution was $0.8 \mathrm{eV}$ for $\mathrm{Al} \mathrm{K} \alpha(1486.6 \mathrm{eV}), 4.5 \mathrm{meV}$ for He II $(40.8 \mathrm{eV})$ and $1.4 \mathrm{meV}$ for He I $(21.2 \mathrm{eV})$ measurements. The sample surface was cleaned by in situ scraping and the cleanliness was ascertained by tracking the sharpness of $\mathrm{O} 1 \mathrm{~s}$ feature and absence of $\mathrm{C} 1 s$ peaks.

Valence band spectra at room temperature obtained using $21.2 \mathrm{eV}, 40.8 \mathrm{eV}$ and $1486.6 \mathrm{eV}$ excitation energies are shown in Fig. 1. There are four discernible features marked by A, B, C and D in the figure. While features B and $\mathrm{C}$ appearing at binding energies (BE) $6>B E>3$ $\mathrm{eV}$ are large in the low excitation energy spectra, the features $\mathrm{A}$ and $\mathrm{D}$ are enhanced significantly in the $\mathrm{Al} K_{\alpha}$ spectrum. The intensity of the feature D becomes maximum in the latter. Considering strong dependence of the relative transition matrix elements on excitation energies [9], the features $\mathrm{B}$ and $\mathrm{C}$ can be attributed to $\mathrm{O} 2 p$ non-bonding electron excitations and the feature $\mathrm{A}$ to the bonding levels with the electronic states having primarily $\mathrm{O} 2 p$ character. The feature $\mathrm{D}$ corresponds to the excitation of primarily Ir $5 d$ electrons.

Since a major conclusion is based on the spectra obtained at low excitation energies, it is important to establish that our conclusions are representative of the bulk. The feature D in Fig. 1 is distinctly separated from the O $2 p$-related signals in the $40.8 \mathrm{eV}$ and $1486.6 \mathrm{eV}$ spectra. Thus, Ir $5 d$ contributions can reliably be delineated by fitting the $\mathrm{O} 2 p$ bands using three Gaussians representing features $\mathrm{A}, \mathrm{B}$ and $\mathrm{C}$ as performed in other systems [10, 11, 12, 13]. The resultant fit obtained by least square error method is shown by lines in Fig. 1 and the extracted Ir $5 d$ band is shown in Fig. 2(a). The spectral intensity at the Fermi level appears to be significantly small in both the spectra, and a peak appears around $1.3 \mathrm{eV}$ binding energy with a tail extending down to $3 \mathrm{eV}$. Interestingly, the spectral lineshape of both $40.8 \mathrm{eV}$ and $1486.6 \mathrm{eV}$-spectra are identical despite their large difference in probing depth; this is demonstrated by superposing the resolution broadened $40.8 \mathrm{eV}$ spectrum (solid line in the figure) over the $1486.6 \mathrm{eV}$-spectrum. This establishes that the surface and the bulk electronic structures are essentially identical in contrast to the observations in $3 d$ and $4 d$ transition metal oxides 10, 11, 12, 13].

We now focus on the evolution of the valence band spectral intensities as a function of temperature in order to investigate the origin of CDW ground state to bring out the point of major emphasis of this paper. No spectral modification is observed down to $183 \mathrm{~K}$. As the temperature is lowered across $183 \mathrm{~K}$, the intensity at $\mathrm{E}_{F}$ in the $40.8 \mathrm{eV}$ spectra decreases dramatically and the leading edge shifts to higher binding energies as shown in
Fig. 2(b). Large resolution broadening in the $1486.6 \mathrm{eV}$ spectra smears out all these changes; however, these spectra ensure that there is no significant modification at higher energy scales. In order to bring out a better clarity, we expand $E_{F}$-region of $40.8 \mathrm{eV}$ spectra in Fig. 2(c) at temperatures $300 \mathrm{~K}, 150 \mathrm{~K}$ and $28 \mathrm{~K}$. The shift of the leading valence band edge to higher binding energy is clearly evident at $150 \mathrm{~K}$, which reduces the spectral weight at $E_{F}$ significantly. A further decrease in temperature leads to an opening of a band gap of the order of $50 \mathrm{meV}$ below $E_{F}$ accompanied by an enhancement in intensity around $0.9 \mathrm{eV}$ binding energy as inferred from Fig. 2(b).

In order to discuss the changes at $E_{F}$ in more detail, we show the high resolution spectra obtained with He I radiations in Fig. 3. The shift in the valence band edge with decreasing temperature is clearly evident in Fig. 3(a). The photoemission response can be expressed as $I(\epsilon)=$ $\int g\left(\epsilon_{1}\right) \cdot F\left(\epsilon_{1}, T\right) \cdot L^{e}\left(\epsilon_{1}, \epsilon_{2}\right) \cdot L^{h}\left(\epsilon_{2}, \epsilon_{3}\right) \cdot G\left(\epsilon_{3}, \epsilon\right) d \epsilon_{1} d \epsilon_{2} d \epsilon_{3}$ where, $g(\epsilon)$ and $F\left(\epsilon_{1}, T\right)$ represent the matrix element weighted density of states and Fermi-distribution function, respectively. The Lorentzian broadenings $\left(L^{e}\right.$ and $\left.L^{h}\right)$ due to the finite lifetime of photo-electrons and photo-holes will be small close to $E_{F}$. Since the resolution broadening represented by the Gaussian, $G$ (full width at half maximum $=1.4 \mathrm{meV}$ ) is negligible compared to the energy scale of investigation, $I(\epsilon) / F(\epsilon, T)$ provide a good representation of the spectral density of states, SDOS, as has also been observed in other systems [12].

The SDOS thus obtained at $300 \mathrm{~K}$ and $150 \mathrm{~K}$ are shown in Fig. 3(b) along with the spectra at other temperatures. It is important to note here that such an estimation of SDOS is sensitive to the precise location of $E_{F}$. Therefore, we have carefully determined $E_{F}$ at each temperature by the Fermi cut off as observed for silver mounted on the sample holder together with the sample. The representative spectra are shown in Fig. 3(a) at $20 \mathrm{~K}$ and $300 \mathrm{~K}$. In order to verify the reliability of our analysis, we carry out the same exercise for silver as described above (see Fig. 3(b)). The room temperature spectrum clearly reproduces the flat density of states of silver in a wide energy range $\left(>3.5 k_{B} T\right)$, thereby providing a confidence in this procedure.

Interestingly, it is clear from Fig. 3(b) that the spectral DOS at room temperature exhibits a finite intensity and a distinct dip at $E_{F}$ suggesting the signature of a 'pseudogap' [7]. This observation in conjunction with the insulating transport behavior [1] suggests that all these electronic states are essentially localized. This is not surprising for such a quasi-one dimensional system where a small lattice distortion and/or impurity leads to localization of the electronic states. Existence of such localized states forming a pseudogap at $E_{F}$ has been predicted long before 7, 8]. High resolution employed for these measurements makes it possible to directly probe such electronic states experimentally. A notable finding is that the in- 
tensity at $E_{F}$ becomes close to zero below the CDW transition, thus forming a soft gap at $E_{F}$. Eventually a gap of the order of $50 \mathrm{meV}$ below $E_{F}$ opens up around $83 \mathrm{~K}$ with a corresponding increase in intensity around $0.9 \mathrm{eV}$ binding energy.

It is well known that the disorder leads to a $\left|E-E_{F}\right|^{1 / 2}$ ( $E$ is energy) cusp at $E_{F}$ in metals [14, 15]. In an insulator consisting of localized electronic states at $E_{F}$, a soft Coulomb gap opens up due to electron-electron Coulomb repulsion; in such a situation, the ground state is stable with respect to a single-particle excitation only if SDOS can be characterized by $\left(E-E_{F}\right)^{2}$-dependence [16, 17]. In contrast to this, in the present case, the SDOS exhibits a $\left|E-E_{F}\right|^{3 / 2}$-dependence spanning a large energy range close to $E_{F}(\mathrm{BE} \leq 300 \mathrm{meV})$ as shown in Fig. 3(c). Most interestingly, this dependence remain unchanged down to the lowest temperature studied. Thus, the opening of a soft gap observed here has an origin different from the electron-electron Coulomb repulsion observed in other systems [17]. The exponent of $3 / 2$ in SDOS suggests strong influence of electron-magnon coupling on the electronic structure [18] presumably responsible for the ferromanetic ground state.

We now turn to another fascinating observation in the core level spectra of metallic ions. In Fig. 4(a), we show Ba $3 d_{5 / 2}$ spectra exhibiting a sharp feature around $779 \mathrm{eV}$ binding energy. Interestingly, a decrease in temperature from $300 \mathrm{~K}$ to $210 \mathrm{~K}$ leads to a significantly large shift in the peak position and there is no further shift as the temperature is lowered further 19]. Similar effect has also been observed for other core levels as shown for Ba $4 d_{5 / 2}$ in Fig. 4(b), while the Ir core levels remain unchanged (see the same figure). The linewidth of the curves for Ba remain the same below $210 \mathrm{~K}$ but it is much larger at room temperature. Interestingly, $300 \mathrm{~K}$ spectra can be reproduced remarkably by a superposition of the $25 \mathrm{~K}$ spectra $(\sim 78 \%)$ and the same shifted by $0.5 \mathrm{eV}(\sim 22 \%)$ towards higher binding energy as shown in the figure. It is therefore clear that the spectra for $\mathrm{Ba}$ at room temperature consists of signals from two types of $\mathrm{Ba}$ ions. However, there are three types of $\mathrm{Ba}$ ions in the crystal structure 3] depending upon the Ba-O bond distances and clustering. We, thus, calculated the Madelung potential considering only the nearest neighbor cluster $\left(V=\sum\left(a / r_{i}\right) ; a\right.$ is a constant). We find that this potential is nearly the same $(\sim 4.05 a)$ for two types of $\mathrm{Ba}$ ions, which is slightly different from the third one $(\sim 4.1 a)$, thereby, qualitatively accounting for the features. In short, the above results reveal that there are profound changes in the covalency effects around $\mathrm{Ba}$ at a temperature higher than the CDW transition temperature. To our knowledge, it is for the first time that such an effect is identified prior to the formation of CDW. While it is not clear whether this is a precursor effect to CDW formation, a confirmation of such a relationship from future studies is expected to open up new direction to understand the origin of CDW, just as pseudo-gap effects in high temperature superconductors have attracted a lot of attention. We hope this work will motivate further work to look for similar features in other non-metals, which will help in the advancement of the knowledge of phase transitions.

In conclusion, to our knowledge for the first time, we have demonstrated directly the evolution of charge density wave due to localized electronic states. It is also shown that a soft gap opens up across the CDW transition. The energy dependence of the spectral density of states reveals the role of magnetism on the electronic structure in the vicinity of Fermi level, which signals an intimate relationship between ferromagnetism and charge density wave in this system. The observation of profound changes in the Ba-O covalency prior to the formation of charge density wave poses a new question with respect to the role of precursor effects.

[1] G. Cao et al., Solid State Commun. 113, 657 (2000).

[2] G. Cao, X.N. Lin, A. Chikara, V. Durairaj, and E. Elhami, Phys. Rev. B 69, 174418 (2004); A. Ohnishi et al., Physica B 329, 930 (2003).

[3] A.V. Powell and P.D. Battle, J. Alloys and Compounds 232, 147 (1996).

[4] R. Lindsay, W. Strange, B.L. Chamberland, and R.O. Moyer, Jr., Solid State Commun. 86, 759 (1993).

[5] B.L. Chamberland, J. Less-Common Met. 171, 377 (1991).

[6] T. Siegrist and B.L. Chamberland, J. Less-Common Met. 170, 93 (1991).

[7] N.F. Mott Metal-Insulator Transitions, 2nd ed. (Taylor \& Francis, London, 1990).

[8] P.W. Anderson, Phys. Rev 109, 1492 (1958).

[9] J.J. Yeh and I. Lindau, At. Data and Nucl. Data Tables 32, 1 (1985).

[10] K. Maiti et al., Europhys. Lett. 55, 246 (2001).

[11] K. Maiti, P. Mahadevan, and D.D. Sarma, Phys. Rev. Lett. 80, 2885 (1998); K. Maiti and D.D. Sarma, Phys. Rev. B 61, 2525 (2000).

[12] K. Maiti et al., Phys. Rev B 70, 195112 (2004).

[13] Kalobaran Maiti and Ravi Shankar Singh, Phys. Rev. B 71 (2005) (MS\# LV9040BR).

[14] B.L. Altshuler and A.G. Aronov, Solid State Commun. 30, 115 (1979).

[15] D.D. Sarma et al., Phys. Rev. Lett. 80, 4004 (1998).

[16] A.L. Efros and B. Shklovskii, J. Phys. C: Solid State Phys. 8, L49 (1975).

[17] J.G. Massey and M. Lee, Phys. Rev. Lett. 75, 4266 (1995).

[18] V.Yu Irkhin, M.I. Katsnelson, and A.V. Trefilov, J. Phys.: Condens. Matter 5, 8763 (1993); V.Yu Irkhin and M.I. Katsnelson, J. Phys.: Condens. Matter 2, 7151 (1990).

[19] A careful look at the first and second derivative of electrical resistivity vs temperature revealed the existence of an anomaly around $250 \mathrm{~K}$ and it is of interest to confirm this on a variety of samples. 


\section{FIGURE CAPTIONS}

Fig. 1 Valence band spectra of $\mathrm{BaIrO}_{3}$ at different excitation energies. The line represents the simulated O $2 p$ contributions using three Gaussians representing features $\mathrm{A}, \mathrm{B}$ and $\mathrm{C}$. The inset shows two $\mathrm{Ir}_{3} \mathrm{O}_{12}$ clusters, which are connected by corner sharing with the cluster long axis tilted by $12^{\circ}$ leading to monoclinic structure.

Fig. 2(a) Ir $5 d$ contributions in $\mathrm{Al} K \alpha$ (open circles) and He II (solid circles) spectra after subtraction of the O $2 p$ features shown in Fig. 1. The solid line through Al $K \alpha$ spectrum, represents the resolution broadened He II spectrum. (b) The temperature evolution of the valence band at $\mathrm{Al} K \alpha$ and He II excitation energies. He II spectra show significant shift of the leading edge at $E_{F}$ with decreasing temperature and the spectral weight appears to be transferred from $E_{F}$ to around $0.9 \mathrm{eV}$ binding energy. (c) Expanded He II spectra close to $E_{F}$ clearly exhibiting the shift of the leading edge across CDW tran- sition. The $25 \mathrm{~K}$ spectrum shows a gap of about $50 \mathrm{meV}$ below $E_{F}$.

Fig. 3(a) High-resolution He I spectra near $E_{F}$. Ag spectra at $20 \mathrm{~K}$ and $300 \mathrm{~K}$ are also shown by stars to precisely determine $E_{F}$. (b) Spectral density of states (SDOS) at room temperature (open circles) and at $150 \mathrm{~K}$ (up-triangles) are compared with the spectra at other temperatures. SDOS for $\mathrm{Ag}$ at $300 \mathrm{~K}$ is shown by stars. (c) SDOS plotted as a function of $(B E)^{1.5}$ show linear dependence.

Fig. 4(a) Ba $3 d_{5 / 2}$ and (b) Ba $4 d_{5 / 2}$ core level spectra at different temperatures. The dashed lines $\mathrm{A}(\mathrm{C})$ and $\mathrm{B}(\mathrm{D})$ in upper(lower) panel are the spectra at $25 \mathrm{~K}$ and the same shifted by $0.5 \mathrm{eV}(0.45 \mathrm{eV})$ towards higher binding energy, respectively. The weighted sum, A+B $(\mathrm{C}+\mathrm{D})$ reproduce the $300 \mathrm{~K}$ spectrum (solid line) remarkably well. Ir $4 f$ spectra collected in the same scan with $4 d$ spectra are shown in (b), which do not show any change with temperature. 


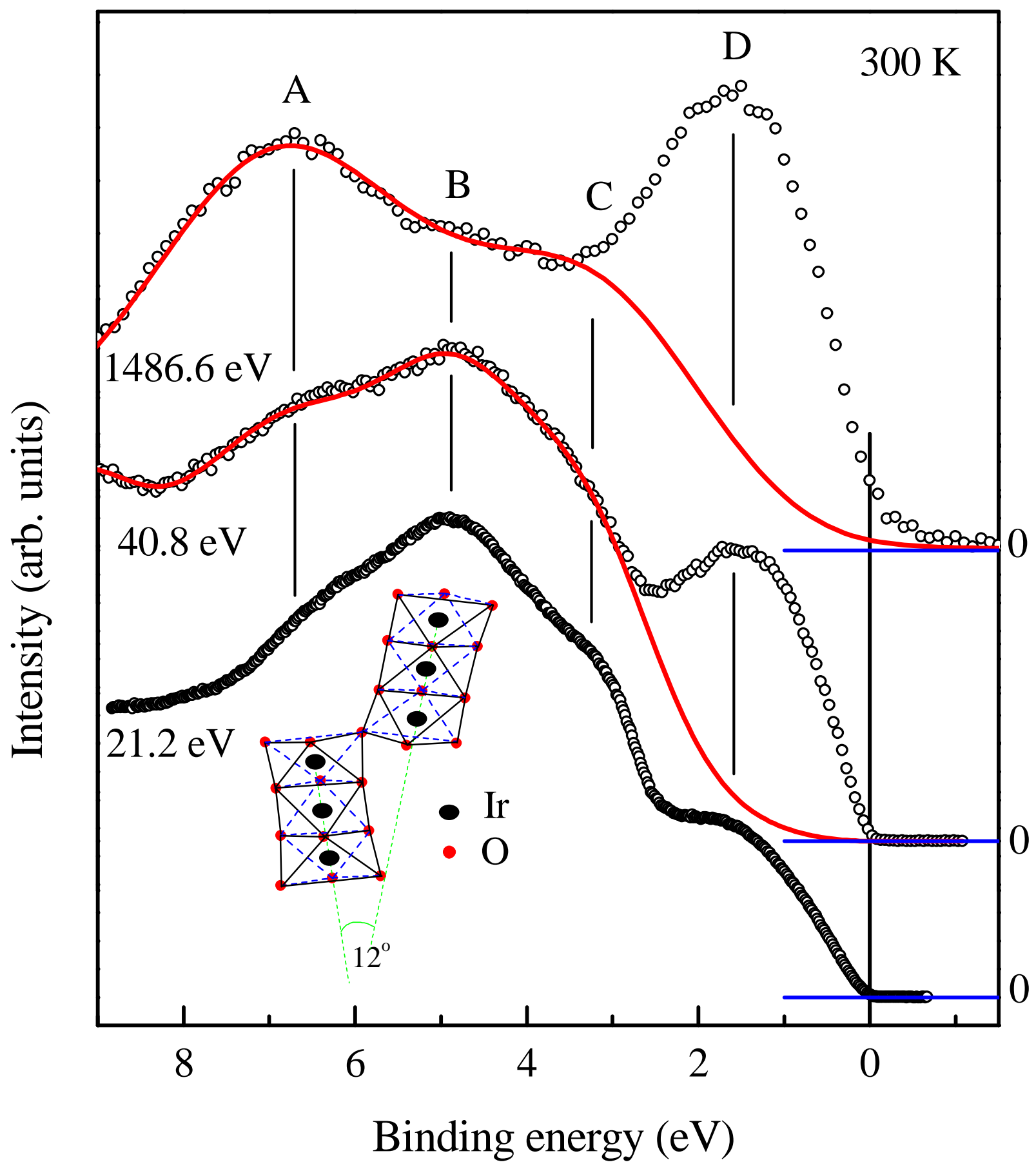

Fig. 1: Maiti et al. 

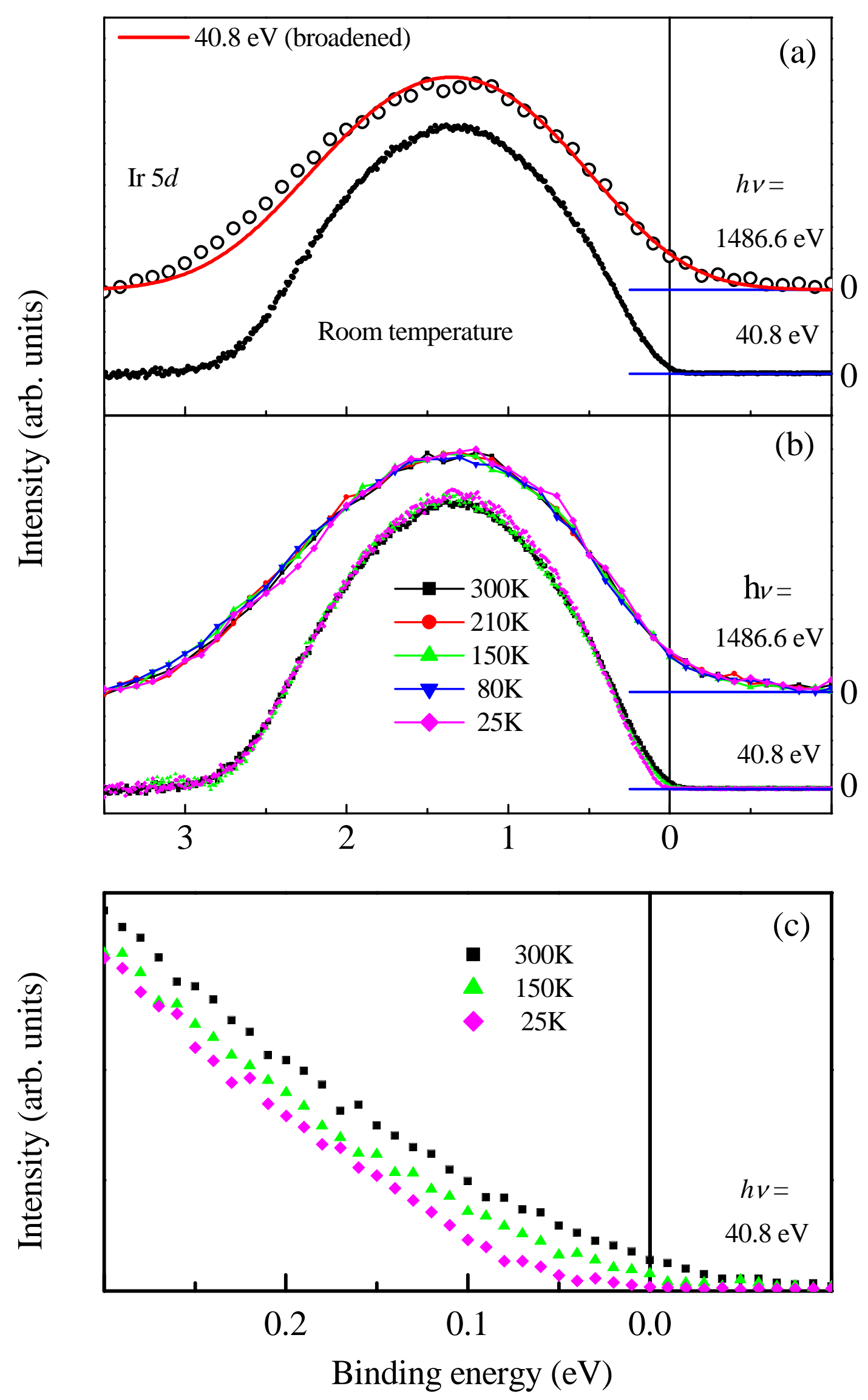

Fig. 2: Maiti et al. 

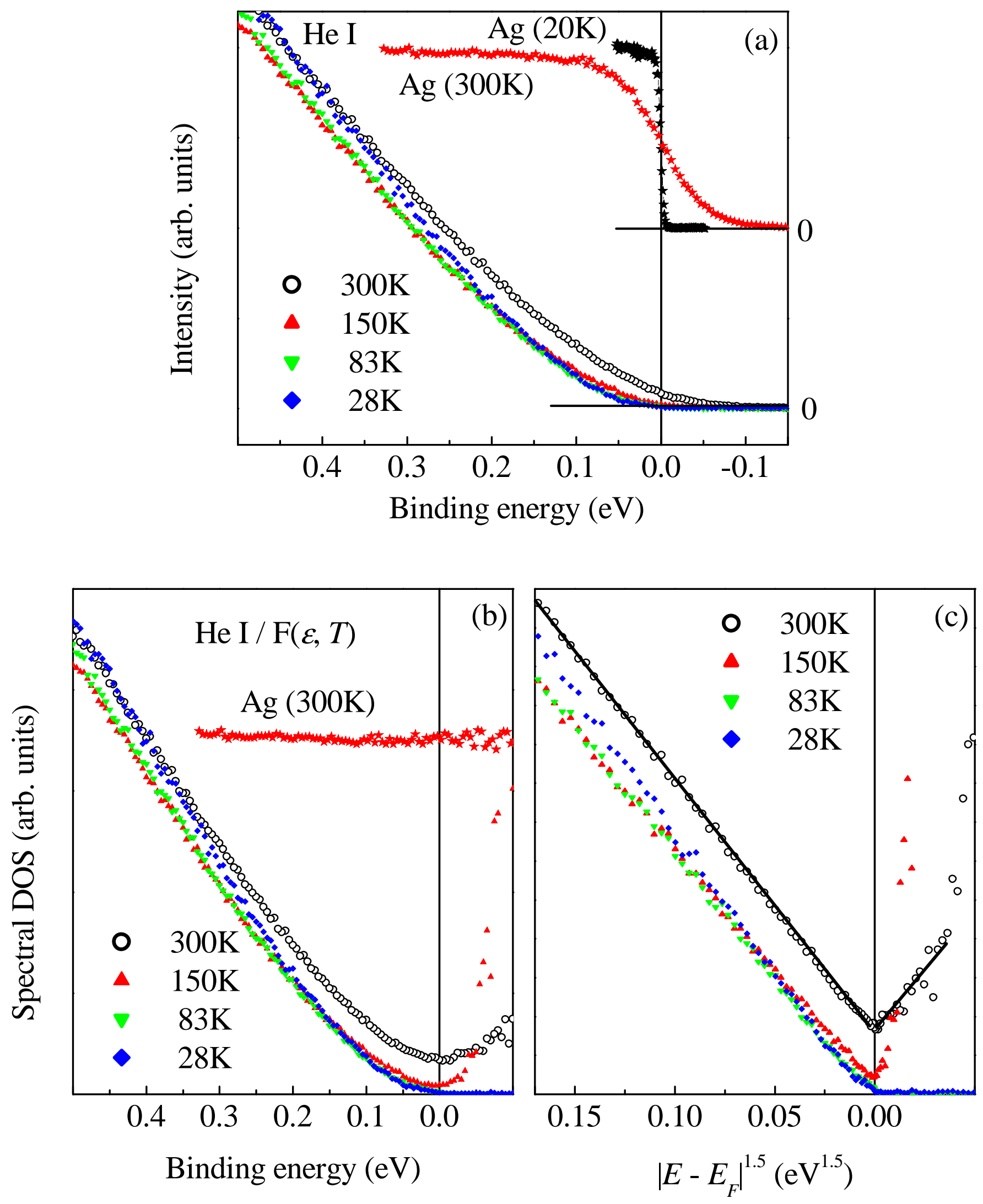

Fig. 3: Maiti et al. 

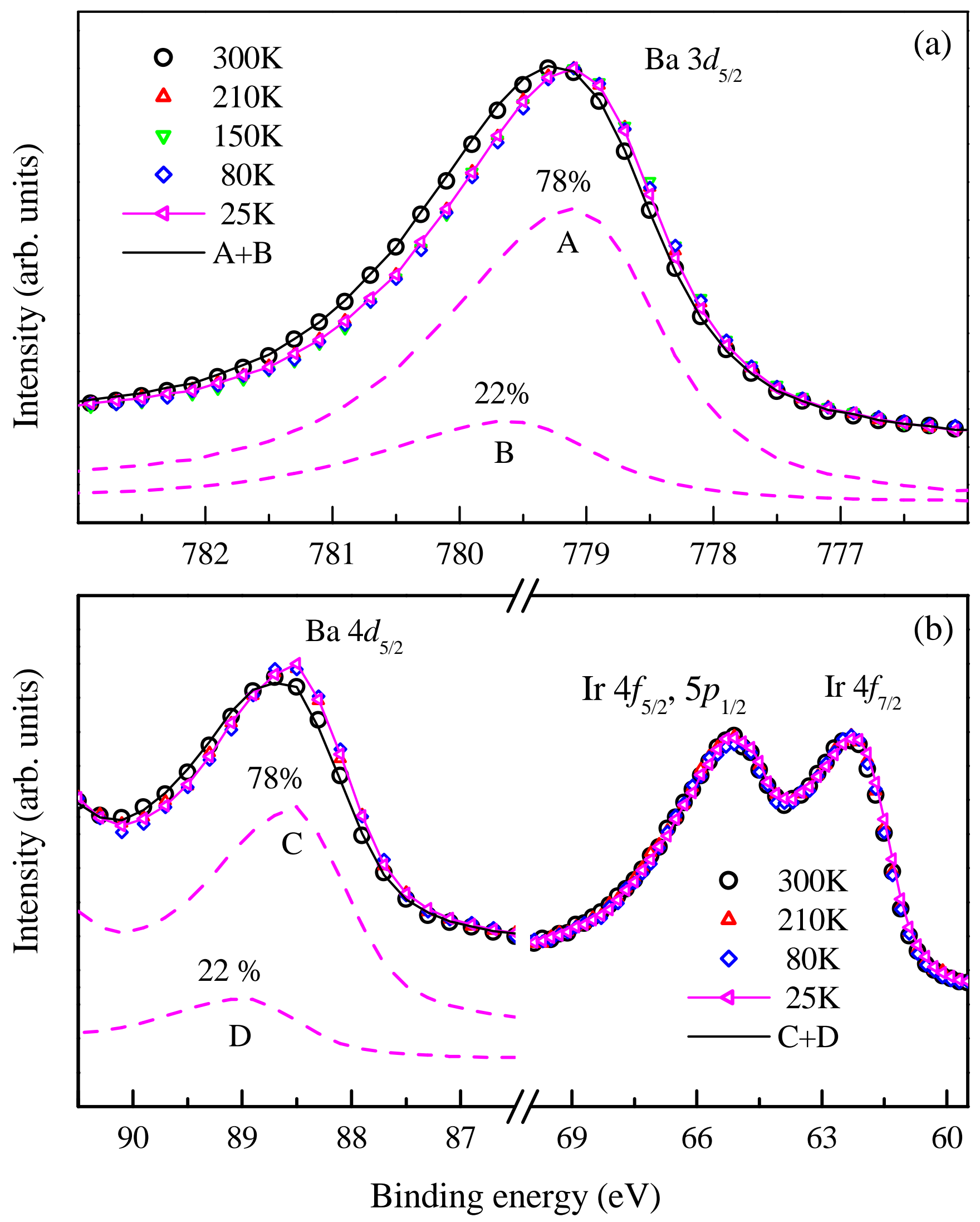

Fig. 4: Maiti et al. 